\title{
RUSSISCH-DEUTSCHER
}

LITERATURTRANSFER DER 30ER-40ER

JAHRE DES 20. JAHRHUNDERTS

This is an open access article distributed under the Creative Commons Attribution 4.0 International (CC BY 4.0)

\author{
(S. TRETJAKOW, F. WOLF, B. BRECHT) \\ (C) 2020. T.A. Sharypina, P.D. Kazakowa \\ Lobachewskij-Forschungsuniversität Nishnij Nowgorod, \\ Nishnij Nowgorod, Russland \\ Datum der Artikelübergabe: I4. Juni 2019 \\ Veröffentlichungsdatum: 25. Juni 2020
}

Inhaltsangabe: Die Auswirkungen des Phänomens der Russischen Avantgarde im ersten Drittel des zwanzigsten Jahrhunderts auf die ethisch-ästhetischen Prinzipien und die Poetik des deutschen sozialistischen Dramas werden analysiert. Ein nationalistischer Mythos wird in Deutschland nach dem Ersten Weltkrieg aktiv gebildet, während die internationale Grundlage in der Literatur Russlands vorherrscht, was für B. Brecht, I. Becher, F. Wolf, E. Weinert konzeptionell attraktiv war. Der Artikel befasst sich mit der Analyse des Einflusses des Theaterstück-Artikels von S. Tretjakow «Brülle, China!» auf das Drama von F. Wolf und B. Brecht. Es wird festgestellt, dass B. Brecht («Der gute Mensch von Sezuan», I938-I94I) in den Traditionen des epischen Theaters versuchte, den Geist des Zuschauers zu wecken und ihm ein anschauliches Beispiel für die Handlung zu geben. Im Stück «Tai Young erwacht» (I93I) nutzt F. Wolf alle Möglichkeiten, um den Zuschauer emotional zu beeinflussen, versucht mit Hilfe der Katharsis, die Mitwirkung des Zuschauers auf der Bühne zu erreichen, und stützt sich auch auf die Traditionen von M. Gorki und A. Fadejew, indem er seine Heldin während des Werdens eines Charakters darstellt. Die Vielfältigkeit der Ansätze zur Aufstellung eines chinesischen Themas in Abhängigkeit von der politischen und ethisch-ästhetischen Ausrichtung der Autoren zeugen von der Produktivität kreativer russisch-deutscher Kulturkontakte.

Stichwort: B. Brecht, F. Wolf, S. Tretjakow, literarischer Transfer, optimistische Tragödie, Katharsis, sozialistische Dramaturgie.

Information zu den Autoren: Tatiana A. Sharypina, Doktor der Philologie, habit., Professor mit Lehrstuhl für ausländische Literatur an der Lobachewskij-Forschungsuniversität Nishnij Nowgorod. Bolshaya Pokrovskaya, 37, 603000 Nishnij Nowgorod, Russland. ORCID ID: 0000-0002-8585-8983

E-mail: swawa@yandex.ru

Polina D. Kazakowa, Kandidat der Philologie, Assistenzprofessor am Lehrstuhl für ausländische Linguistik an der Lobachewskij-Forschungsuniversität Nishnij Nowgorod. Bolshaya Pokrovskaya, 37, 603000 Nishnij Nowgorod, Russland.

E-mail: polina_iwliewa@mail.ru

Zum Zitieren: Sharypina T.A., Kazakowa P.D. Russisch-Deutscher Literaturtransfer Der zoer4oer Jahre Des 20. Jahrhunderts (S. Tretjakow, F. Wolf, B. Brecht). Studia Litterarum, 2020, vol. 5, no 2, pp. I46-I6I. (In German) DOI: IO.22455/2500-4247-2020-5-2-I46-I6I 


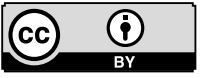

This is an open access article distributed under the Creative Commons Attribution 4.0 International (CC BY 4.0)

\section{RUSSIAN-GERMAN LITERARY TRANSFER OF THE 1930S-40S (S. TRETYAKOV, F. WOLF AND B. BRECHT)}

\author{
(C) 2020. T.A. Sharypina, P.D. Kazakova \\ Lobachevsky State University of Nizhni Novgorod, \\ Nizhni Novgorod, Russia \\ Received: June I4, 2019 \\ Date of publication: June 25, 2020
}

Abstract: The essay examines the influence of Russian literature upon ethical and aesthetic principles and poetics of German socialist drama. After the defeat in the WWI, Germany developed nationalist myths, whereas the international principle prevailed both in Russian literature and culture. The latter fact attracted B. Brecht, J. Becher, F. Wolf, and many others. The essay's aim is to demonstrate the influence of the playarticle Roar China! A Drama in Seven Scenes by S. Tretyakov on the German socialist drama. It is stated that Brecht (The Good Person of Szechwan, I938-I94I), considering the traditions of the epic theater, tried first of all to awaken the mind of the spectator and to give a clear example of action. In the play Tai Yang erwacht (I93I), F. Wolf used all available means of impact to influence the viewer emotionally, aiming to achieve the involvement of the audience into what was going on the stage by means of catharsis, and within the traditions of M. Gorky and A. Fadeyev depicted his heroine in the process of her character development. The versatility and the multidimensional nature of approaches to the development of the Chinese theme is the evidence of the productivity of artistic Russian-German cultural contacts.

Keywords: B. Brecht, F. Wolf, S. Tretyakov, literary transfer, optimistic tragedy, catharsis, socialist drama.

Information about the author: Tatiana A. Sharypina, DSc in Philology, Professor, Head of the Department of Foreign Literature, Lobachevsky State University of Nizhni Novgorod, Bolshaya Pokrovskaya St. 37, 603000 Nizhny Novgorod, Russia. ORCID ID: 0000-0002-8585-8983

E-mail: swawa@yandex.ru

Polina D. Kazakova, PhD in Philology, Assistant Professor of the Department of Foreign Linguistics, Lobachevsky State University of Nizhni Novgorod. Bolshaya Pokrovskaya St. 37, 603000 Nizhny Novgorod, Russia.

E-mail: polina_iwliewa@mail.ru

For citation: Sharypina T.A., Kazakova P.D. Russian-German Literary Transfer of the I930s-4Os (S. Tretyakov, F. Wolf, and B. Brecht). Studia Litterarum, 2020, vol. 5, no 2, pp. I46-I6I. (In German) DOI: I0.22455/2500-4247-2020-5-2-I46-I6I 
УДК 82I.II2.2 + 82I.I6I.I

ББК $83.3(4$ Гем $)+$

$83.3(2 \mathrm{Poc}=\mathrm{Pyc})$

\section{РУССКО-НЕМЕЦКИЙ ЛИТЕРАТУРНЫЙ TРАНСФЕР 30-Х-40-Х ГГ. ХХ В. \\ (С. ТРЕТЬЯКОВ, Ф. ВОЛЬФ, Б. БРЕХТ)}

(C) 2020 г. Т.А. Шарыпина, П.Д. Казакова

Национальный исследовательский Нижегородский государственный университет им. Н.И. Лобачевского, Нижний Новгород, Россия

Дата поступления статьи: г4 июня 2020 г.

Дата публикащии: 25 июня 2020 г.

DOI: I0.22455/2500-4247-2020-5-2-I46-I6I

Аннотация: Анализируется влияние феномена русского авангарда первой трети $\mathrm{XX}$ в. на этико-эстетические принципы и поэтику немецкой социалистической драматургии. В Германии после Первой мировой войны активно формируется националистический миф, тогда как в литературе России преобладало интернациональное начало, что было концептуально притягательно для Б. Брехта и Ф. Вольфа. Целью статьи является анализ влияния на драматургию Ф. Вольфа и Б. Брехта пьесы-статьи С. Третьякова «Рычи, Китай!». Констатируется, что Б. Брехт («Добрый человек из Сычуани», I938-І94I) в традициях эпического театра пытался пробудить разум зрителя и дать наглядный пример действия. В пьесе «Тай Янг пробуждается» (I93I) Ф. Вольф использует все способы эмоционального воздействия на зрителя, при помощи катарсиса старается добиться сопричастности зрителей происходящему на сцене, а также опирается на традиции М. Горького и А. Фадеева, изображая свою героиню в процессе становления характера. Многоплановость подходов к разработке китайской темы в зависимости от политической и этико-эстетической ориентации писателей свидетельствуют о продуктивности творческих российсконемецких культурных контактов.

Ключевые слова: Б. Брехт, Ф. Вольф, С. Третьяков, литературный трансфер, оптимистическая трагедия, катарсис, социалистическая драматургия.

Информация об авторах: Татьяна Александровна Шарыпина - доктор филологических наук, профессор, заведующий кафедрой зарубежной литературы, Национальный исследовательский Нижегородский государственный университет им. Н.И. Лобачевского, ул. Большая Покровская, д. 37, 603000 г. Нижний Новгород, Россия. ORCID ID: оооо-о0о2-8585-8983

E-mail: swawa@yandex.ru

Полина Дмитриевна Казакова - кандидат филологических наук, доцент кафедры зарубежной лингвистики, Национальный исследовательский Нижегородский государственный университет им. Н.И. Лобачевского, ул. Большая Покровская, д. 37, 603000 г. Нижний Новгород, Россия.

E-mail: polina_iwliewa@mail.ru

Для цитирования: Шарыпина Т.А., Казакова П.Д. Русско-немецкий литературный трансфер 30-х-40-х гг. ХХ в. (С. Третьяков, Ф. Вольф, Б. Брехт) // Studia Litterarum. 2020. T. 5, № 2. C. I46-I6I. DOI: IO.22455/2500-4247-2020-5-2-I46-I6I 
Der Prozess der russisch-deutschen literarischen und kulturellen Wechselbeziehungen und der des Transfers von philosophischen, ästhetischen, künstlerischen Ideen und Erscheinungen ist der Gegenstand intensiver Forschung sowohl in der deutschen als auch in der russischen Literaturwissenschaft seit Jahrzehnten [I8; 19; 20; 2I]. Es gibt jedoch immer noch Fragen, die in den wissenschaftlichen Arbeiten nicht genug ausreichend behandelt wurden, darunter auch das Problem des transnationalen Phänomens der Avantgarde im ersten Drittel des 20. Jahrhunderts. Die meisten deutschen Avantgarde-Künstler stammen aus der «Schule» des Expressionismus, in deren Manifesten eine internationale und zeitlose Beschaffenheit mehrmals betont wurde [9, S. 3IO-3II]. Nach dem Ersten Weltkrieg begann der germanisch-nationalistische Mythos in der soziokulturellen Atmosphäre Deutschlands zu bilden, während die internationale Grundlage in der Literatur und Kultur Sowjetrusslands vorherrschte, was für B. Brecht, I. Becher, F. Wolf, E. Weinert konzeptionell attraktiv war. Das internationale Fabel- und Themenspektrum war für deutsche sozialistisch orientierte Schriftsteller grundlegend. Friedrich Wolf, einer der bekanntesten Vertreter des deutschen sozialistischen Dramas des 20. Jahrhunderts, erklärte in seiner Rede auf dem Ersten Kongress der sowjetischen Schriftsteller, dass die Fragen der Existenz und Entwicklung der deutschen antifaschistischen Literatur nicht ausschließlich national sind, sondern sie sind von entscheidender Bedeutung für die Stärkung der fortschrittlichen Schriftsteller der ganzen Welt im Kampf gegen Faschismus. Sergei Tretjakow, ein brillanter Publizist, ein futuristischer Dichter (einer der Leiter der LEF-Gruppe), ein innovativer Dramatiker und Übersetzer, dessen Bemühungen im Vorkriegsjahrzehnt eine bedeutende Rolle bei der Stärkung der sowjetischen und deutschen sozialistisch orientierten Schriftsteller spielten, widmete diesem 
Thema eine eindrucksvolle pathetische Rede. S. Tretjakow erinnert sich unter anderem an seine Kontakte zu B. Brecht. Ein anschauliches Beispiel für den Kulturtransfer Russland-Deutschland ist die Verkörperung des Themas des rebellischen China.

Die Bedeutung der chinesischen Themen in der europäischen Literatur und Kultur in den späten zoer und frühen zoer-4oer Jahren des zwanzigsten Jahrhunderts wurde von den sozialen und politischen Prozessen geprägt, die dieses große Land auf dem Weg der Selbstbestimmung im Kampf um nationale Unabhängigkeit umfasst haben. Die aktuelle Situation des Kulturtransfers unterscheidet sich von der Aktivierung des Interesses an China in den zwanziger und vierziger Jahren des zwanzigsten Jahrhunderts. Um die Transformation der gegenseitigen Wahrnehmung in der Dynamik des literarischen Prozesses an der Wende des XX-XXI Jahrhunderts richtig zu verstehen, muss man den literarischen Transfer der 3oer-4oer Jahre gründlich untersuchen. Dieser Transfer «Russland - Deutschland - China» hatte nämlich meist eine schwierige Form. Somit entstanden zu dieser Zeit in Russland Gedichte über China von Wladimir Majakowski wie «Moskau China», «Finger weg von China!» und «Lies mal und ab nach Paris und China». Dazu gehören auch Gedichte von Demjan Bedny. In ihren Kunstwerken drückten die Schriftsteller ihr Mitgefühl für unterdrückte Menschen in China aus und prangerten die Militär Diktatur der Kuomintang an, die sich vom Westen mit Schätzen ihres Landes freikaufen wollte. In dieser Zeit erscheinen ferner eine Erzählung von Nikolai Tichonow über Sun YatSen, ein Gedicht von Saken Seifullin sowie seine Dokumentarfilme «Der große Flug» (1925) und «Schanghaier Tagebuch». Im Jahre I925 wurde das Ballett von Reinhold Gli re «Rote Mohnblume» inszeniert. Einen besonderen Einfluss auf europäische namentlich deutsches sozialistisches Drama übte Theaterstück Tretjakows «Brülle, China!» (I924) aus, das sich durch eine überaus markante Poetik auszeichnete. Es war das Ergebnis seines Aufenthalts in China und wurde im Meyerhold Theater von W. Fedorov unter Leitung von Meyerhold inszeniert. Später schrieb S. Tretjakow ein ergreifendes Gedicht «Brülle, China!», das ein «poetisches Gelübde darstellt. Ein Gelübde das zur Rache für die Demütigung eines großen Volkes aufruft» [5, S. 554-564]. Das Spiel fand großen Zuspruch und wurde im Jahre 1929 in Deutschland in Frankfurt am Main uraufgeführt. Die österreichische Tageszeitung «Arbeiter-Zeitung» hat das Spiel auf die gleiche Stufe mit «Panzerkreuzer Potemkin» von Sergei Eisenstein gestellt. 
Im Kontext der soziokulturellen Situation 30-40er und des allgemeinen Interesses der europäischen Kunst an der Kultur und der politischen Situation in China stellt die Vergleichsanalyse der ideologischen und künstlerischen Struktur der dramatischen Werken von F. Wolf («Tai Yang erwacht», I93I) und von B. Brecht «Der gute Mensch von Sezuan», (I938-I94I) ein riesiges Forschungsdesiderat dar. Die Vergleichsanalyse ermöglicht, die unterschiedlichen Herangehensweisen festzustellen, mit denen das chinesische Thema und der Hauptkonflikt der Werke verwirklicht werden, und das vor allem im Hinblick auf Zugehörigkeit zum politischen (F. Wolf) beziehungsweise epischen Theater (B. Brecht).

Künstlerisches Schaffen von Friedrich Wolf (Friedrich Wolf, I888-I953) nimmt zu Recht einen bedeutsamen Platz in der deutschen Literatur des zwanzigsten Jahrhunderts ein. Neben B. Brecht war F. Wolf der größte Dramatiker der sozialistischen Richtung der 30-40er Jahre, der zum Teil die Weiterentwicklung des nicht nur deutschen, sondern auch des gesamteuropäischen Theaters vorausbestimmt hat.

F. Wolf betritt die Weltbühne mit Stücken «Zyankali», I929 und «Professor Mamlock», I933; den Wendepunkt erfährt sein Schaffen aber bereits im Jahr 1923 mit dem Stück «Der Arme Konrad». Während der Arbeit an dem Stück «Der Arme Konrad» findet Wolf sein allgegenwärtiges Thema - das Thema des Volksaufstands, das mit den Motiven der Abfolge von revolutionären Ideen und denen der politischen Reife der Massen im Kampf um ihre Rechte aufs Engste verbunden ist. Dies wird zum zentralen Thema im Stück «Tai Yang erwacht». Solch ein Bühnenwerk Wolfs lässt sich als «optimistische Tragödie» bezeichnen, in deren Mittelpunkt die Einsichtskatharsis steht. Im Jahre 1926 veröffentlichte der Schriftsteller einen Programmartikel «Der Dichter und das Zeitgewissen». Den Dichter sieht Wolf als Zeitgewissen, der hart und unparteiisch das Zeitalter spiegelt. Sein ästhetisches Credo definiert Wolf als «Kunst ist Waffe», und begründet dieses theoretisch in der gleichnamigen Broschüre im Jahre I929. In seinen Beiträgen, Essays, Artikeln und Gedichten appelliert Wolf immer wieder an Künstler, auf aktuelle Ereignisse scharf zu reagieren. Eine unerschöpfliche Ideenquelle dafür seien die Hektik des Alltags, Streiks, Zeitungen, Statistiken und Tatsachen. Als überzeugter Internationalist ist Wolf auf der leidenschaftlichen Suche nach neuen Formen der Verkörperung vom politischen Material und neuen Wegen, auf den Zuschauer zu wirken. Dabei wäre zu betonen, dass Wolf es sich vornimmt, auf den Zuschauer emotional zu wirken und mithilfe der Katharsis den Zuschauer 
zum Miterleben zu bringen. Brecht hingegen hat es sich angelegen sein lassen, vor allem den Geist zu wecken und ein anschauliches Beispiel für die Aktion zu geben. F. Wolf versucht sich in verschiedenen Genres und Themen, wobei er seinen Sujets prinzipiell internationalen Charakter zugrunde legt. So widmet er ein Hörspiel mit dem Titel «Krassin rettet Italien» der Rettung der Nobile-Nordpolexpedition. Weiterhin spiegelt sich in Wolfs «Marsch auf Mossul» der Kampf des Westens um Bodenschätze des Nahen Ostens wider. I93I erscheint das Drama «Tai Yang erwacht», das von der für Wolf typischen aufrichtigen Utopie geprägt ist. Da der Dichter unentwegt bestrebt ist, den Weg der Einsicht und den der politischen Reife der Arbeiterbewegung im Kampf um ihre Rechte zu zeigen, mutet die Umwandlung des Protagonisten teilweise nicht authentisch, ja gekünstelt an. Thema und Problematik sowie stilistische Ausdruckskraft des Dramas «Tai Yang» stehen offensichtlich im Einklang mit Ideen und Bildern des Theaterstücks und gleichnamigen Dramas «Brülle, China!» von Tretjakow.

Die 1930, I947 und 195I veröffentlichten Spiele enthalten ein Vorwort, das über historische Ereignisse berichtete, z.B. über ausbeutende Kinderarbeit in Schanghai von über hunderttausend Kindern oder darüber, dass der Monatslohn von Weberinnen nur 9 Mexikanischer Dollar betrug, während das Existenzminimum für eine Familie bei 30 Dollar lag. F. Wolf deckt weitere erschreckende Tatsachen auf, dass die Hände der Weberinnen bei kleinsten Fehlern zur Strafe mit kochendem Wasser verbrüht wurden. All diese unbequemen Tatsachen kommen zum Ausdruck in Wolfs Spielen, was ihnen noch mehr Aussagekräftigkeit verleiht. Doch auch an dichterischer Bildhaftigkeit lässt sich das Vorwort es nicht ermangeln. Beispielsweise bezeichnet Wolf chinesische Demonstrationen und Meutereien als «ein Sichrecken halb schlummernden gefesselten Riesen» [23, S. 97], der sich nunmehr, also I947, auf die Beine gestellt hat. I95I stellte der Autor mit Stolz fest, dass «Tai Yang» der Vorbote des zum neuen Leben erwachten chinesischen Volks war.

Trotz des dokumentarischen Charakters des Bühnenwerks, der sich in Gesprächen oder Anmerkungen oder Diskussionen zeigt und vom Vorwort antizipiert wird, spielt sich die Handlung durchaus rasch ab. Das ist unter anderem der besonderen Gliederung des Spiels zu verdanken. Das Stück ist nicht in Akte, sondern in Bilder gegliedert; jedes Einzelne davon zeichnet sich durch hohe Dynamik aus. All dies sorgt für die Atmosphäre der Erwartung, des Anbruchs einer neuen Epoche und allgemeiner Veränderung. Um die notwendige Atmosphäre aufrecht- 
zuerhalten, führt F. Wolf darüber hinaus in seine Spiele eigenartige volkstümliche Lieder ein, die chinesische Volkslieder nachahmen sollen.

Eines, in dem sich um das Übel der Vorgänger handelt, eröffnet den Akt wie folgt: «Bambus flattert und Wolke schiebt, // Blutt rollt und Welle stiebt, // Alles schaukelt wie eine Affenherde... // Still liegt am Ahnenberg Yu-Chan // Die Erde // Man muss wissen, wo man hingehört» [23]. Dasselbe Ziel wird auch durch das Leitmotiv, das Lied über den Mangobaum, verfolgt. Dessen eingangs langsamer Rhythmus symbolisiert die unveränderbare, gleichmäßige Lebensordnung in China, die seit Jahrtausenden von Bestand ist. «Viele tausend Jahre steht der Mangobaum, // Tausend Menschen lagen in seinem Schatten, // Überhundert Geschlechtern // Rauscht der Mangobaum» [23]. Nähert sich das Stück seiner Mitte, ändert sich langsam der gleichmäßige Rhythmus: Zunächst einmal aus dem Munde eines Arbeiters Wan entwichen, ertönt das Lied schließlich als Kampflied des zum Aufstand bereiten chinesischen Volkes: «Einmal aber fährt ein Sturm in den Mangobaum, // Einer der Äste saust nieder,ward zum Speer, // Einer ins Feuer fiel, ward zur Jackel, // Einer zerschlug, ward der Schaft zum Gewehr, // Hundert Gewehre, tausend Gewehre, zehntausend Gewehre // Aus dem uraltem Mangobaum» [23, S. I86].

Derlei Technik findet übrigens in Hauptmanns Drama «Die Weber» Anwendung, das sich ebenfalls dem Aufstand des Volks widmet, das unter unmenschlichen Arbeitsbedingungen richtiggehend darbt und leidet. Hier lässt sich also eine gewisse Tradition erahnen. F. Wolf macht auch zum Leitmotiv das sich im Laufe des Stücks verwandelnde Lied. Das nationale Kolorit des Stücks hängt mit der Parabel über zwei Blumen zusammen, die auf einem blutroten Mohngefilde wachsen, das zwar den Menschen einen Opiumrausch verbürgt und den Alltag vergessen lässt, allerdings Kriege, Wahn und Zwist mit sich bringt, was vom Westen herbeigesehnt wird. Es steht mittlerweile fest, dass Schlafmohn nach China von Engländern eingeführt wurde. Das Rauschgift hielt Einzug ins Leben ärmerer Bevölkerung und berauschte bisweilen ganze Siedlungen [8]. Dem Mohn wird eine kleine weiße Baumwollpflanze gegenübergestellt, die im Herbst aufblüht und den Fleiß der Chinesen symbolisiert. Sie bietet ihnen Arbeit, Bekleidung und Nahrung; daraus wird Papier hergestellt, auf dem man die Wahrheit über das Leben eines Volks und Flugblätter schreiben kann. Die Protagonistin ist noch weit entfernt von der Offenbarung, doch die Parabel, die ihr Arbeiter Wan erzählt, flößt ihr Angst ein; Angst vor etwaigen Streikwellen und Demons- 
trationen, die zu Krawallen und Blutvergießen führen werden. Das Hauptthema dieses Stücks, wie auch vieler anderen Wolfs, ist das der Offenbarung und des Werdegangs des Charakters vom Protagonisten. Gegebenenfalls geht es um ein chinesisches Mädchen namens Tai Yang. Also kein Wunder, dass Wolf Alexander Fadejews Roman «Die Niederlage» besonders wertschätzte. Denn als Dramendichter fühlt sich Wolf besonders davon angesprochen, dass Fadejew den Durchbruch vom Lewinsons Partisanenverband als Werdegang des Charakters schildert [2, S. 35I]. Es vergeht eine Weile, bis Tai Yang eine Offenbarung erlebt. Zunächst einmal erfolgt mithin keine Offenbarung, als Tai Yangs zwölfjährige Schwester Ma als Strafe für einen zerrissenen Faden vom Fabrikherrn Tschu Fu missbraucht werden soll. Das Geschehen hält Tai Yang sogar für ein Glück für sie und ihre Familie. Doch der Zucker in der Zuckerdose, wie das ironisch in der Fabrik bezeichnet wird, erweist sich als ziemlich bitter: Genau auf Tschu Fus Befehl hin werden die Fabrikarbeiter misshandelt und genau seinetwegen verrät Tai Yang, wenn auch im Unwissen, ihren Geliebten Wan. Auf den ersten Blick scheint Tai Yang, ganz unversehens die Offenbarung zu erleben. Indessen wird der Zuschauer durch einzelne Züge und Anmerkungen an den Umbruch im Sinn und Geist der Figur herangeführt. Etwas geradliniger und weniger begründet ist die Offenbarung der Fabrikarbeiterinnen ausgefallen. Eine besondere Rolle im Stück kommt Bildern 7 und $8 \mathrm{zu}$, in denen sich Tai Yang dazu entschließt, Flugblätter in der Fabrik zu verteilen. Unerkennbar gekleidet, verteilt sie nicht nur die Flugblätter, sondern auch rote Tücher, die Solidarität mit den Streikenden symbolisieren. Daher kommt man nicht um die Gedanken herum an den Roman Maxim Gorkijs «Die Mutter», der von Wolf überaus begehrt wurde. In Wolfs Drama vermitteln keine Figuren einen gestelzten noch gekünstelten Eindruck. Sowohl Tai Yang, als auch die Fabrikarbeiterinnen sind nicht ideal: Erst zum Schluss des Stücks erlebt die zwölfjährige Ma die Offenbarung, die bislang überzeugt ist, dass ihre Schwester selbst an die Zuckerdose heranwollte; ihr älterer Bruder Tse ist Aufseher in der Fabrik, ihre Urgroßtante Tse-Tse verkauft Opium. Dagegen weist die Figur Tschu Fus durchaus positive Züge auf. Nur die Figuren der westlichen Missionare und der Rotes-Kreuz-Delegierten Miss Lund sind grotesk gehalten. Es ist bemerkenswert, dass genauso in Hauptmanns «Die Weber» das Stück an dem Höhepunkt abbricht, als die aufständischen Weber das Militär vertreiben. Damit schafft Hauptmann eine eigenartige Hymne für das meuternde Volk. Wolf beendet sein Stück ebenfalls symbolisch: Mit einem Lied über den Mangobaum, das 
Symbol Chinas, das Wanderern Rast und Ruhe bietet und aus dessen Zweigen die Aufständischen im Kampf um ihre Freiheit Waffen anfertigen.

Angesichts der politischen und kulturellen Situation der 1920-30er Jahre erfuhr das chinesische Thema damals großen Zulauf. Annähernd zu dieser Zeit arbeitet Wolfs dramaturgischer Rivale Bertolt Brecht an seinem Stück «Der gute Mensch von Sezuan», 1938-I94I. Desgleichen schildert das Stück einen steinigen Weg der Offenbarung der Protagonistin, die indes im Gegensatz zu Tai Yang nicht über den Scheideweg hinaus gelangt. Zur gleichen Zeit arbeitet Brecht schon seit Jahren an dem Prosabuch «Me-ti. Buch der Wendungen», das in chinesischer Manier verfasst ist und in bester Tradition von M. de Montaigne, J. Swift und F. Bacon. Der Titel stellt den verballhornten Eigennamen des altchinesischen Philosophen und Politikers Mozi und seines Werks «Buch der Wendungen». Die chinesisch stilisierten Versuche Brechts bestehen zwar aus fünf Bänden von Parabeln und Aphorismen, werfen aber die akutesten Probleme der 20-40er Jahre auf, darunter Aufarbeitung und Revision des Marxismus. Erst nach dem Tode Brechts wurde es möglich, das Buch herauszugeben. Für das Stück «Der gute Mensch von Sezuan» wählt Brecht eine sehr markante Form, die zum einen bedingt märchenhaft, zum anderen sinnlich-konkret ist. Den Anstoß dazu nahm er aus der Ballade «Der Gott und die Bajadere» von J.W. von Goethe. Wie so oft schon «verfremdet» Brecht ein bekanntes Sujet, indem er folgende Fragen zu Tage fördert: Ob die Bajadere die Vergebung Gottes überhaupt braucht und ob es wahrlich schwer ist, im Himmel gut zu sein, oder aber es wichtiger wäre, auf dieser Erde gut zu bleiben. Des Problems nimmt sich der Dramatiker dialektisch an, wobei eine paradoxale Gesellschaft geschildert wird, in der das Gute zum Bösen wird und nur dank des Bösen das Gute existieren kann. Dergleichen Probleme der Ethik beschäftigten den Schriftsteller seit je her. Als er seine früheren Werke kommentierte, behauptete er beispielshalber, dass es unmöglich ist, in einer konkreten Gesellschaft nach abstrakten ethischen Vorstellungen zu leben: «Freiheit, Güte, Gerechtigkeit, Geschmack und Großzügigkeit sind Produktionsfragen, sagte Me-ti zuversichtlich <...>. Unter sittlichem Verhalten kann ich nur ein produktives Verhalten verstehen. Die Produktionsverhältnisse sind die Quellen aller Sittlichkeit und Unsittlichkeit» [7, S. 84]. Aus «Dem Versuchsbuch» wird ersichtlich, dass der Autor mit den früheren Ideen Lenins vertraut war, die besagten, dass es im ganzen Marxismus kein Gran Ethik ist. Me-ti, Brechts Alter Ego, macht darauf aufmerksam, dass weder Ka-Meh (Karl Marx) noch Mie-en leh (Lenin) 
eine Sittenlehre vorschlägt. Doch auch hier ist Brechts Selbstständigkeit nicht zu übersehen. Man kommt nicht umhin, S.N. Semljanoj in dem Punkt zuzustimmen, dass Bertolt Brecht nach der Verkörperung seiner eigenen ethischen Utopie trachtete. Er träumt von dem Land, das von seinen Einwohnern keine besondere Tugendhaftigkeit verlangen und darum keine vorgeschriebene Ethik benötigen würde [6, S. 45-46].

Die chinesische, fast märchenhaft anmutende Entourage darf der Zuschauer aber nicht für bare Münze nehmen: Hier liegt ein Gesamtbild eines Staates vor, in dem das Gesetz der sozialen Ungerechtigkeit herrscht. Die kläglichen Bedingungen, unter denen eine junge, arme Prostituierte Shen Te dahinlebt, haften jedweder Gesellschaft der Unterdrückung an. Die Tatsache, dass die Handlung nach China verlagert wird, verleiht dem Stück einen überzeitlichen Charakter sowie «den Verfremdungseffekt». Der Begriff der Verfremdung hat mit dem der Entfremdung von Karl Marx nichts gemeinsam und entstammt dem literarischen Konzept der Verfremdung von Wiktor Schklowski, den Bertolt Brecht über Sergej Tretjakow kennengelernt hat. Die chinesische Entourage, wie sie in Brechts «Buch der Wendungen» und «Der gute Mensch von Sezuan» vorzufinden ist, ist somit keine Nachahmung noch Stilisierung, sondern eine Verfremdung, von der Brecht weiterhin in seinem nicht beendeten, ebenfalls in China spielenden Roman über TUI (Intellektuelle) Gebrauch macht. Man geht wohl nicht fehl in der Annahme, dass Brecht nicht nur das Sujet der Ballade von Goethe verfremdet, sondern er ruft auch die Folkloretradition eines wunderlichen Märchens ins Gedächtnis, wo ein gutes aber von bösen Menschen beleidigtes Waisenkind von der Glücksfee belohnt wird. Auf der Suche nach wenigstens einem guten Menschen führen die Götter einen regelrechten sozialen Versuch am Schicksal Shen Tes durch, in dem sie aber zu deren Unzufriedenheit durchfällt. Denn im Staat, wo die Ungerechtigkeit ungeheuer wuchert, gelten die Märchenregeln nicht länger. Für das Geld, das Shen Tes von den Göttern bekommt, eröffnet sie einen Tabakwarenladen und hilft allen Notleidenden. Doch ein paar Tage später wird ihr klar, dass sie böse werden soll, um weiter Gutes zu tun. So erblickt das Licht der Welt ihr Cousin, der böse und eigennütziger Shui Ta. Langsam leuchtet es Shen Te ein, dass sie nur dann überleben kann, wenn sie sich an den Prinzipen ihres Cousins hält und lediglich von Zeit zu Zeit auch gut ist. Wie paradoxal das auch immer bedeuten mag. Brecht hielt das für vollkommen inakzeptabel, wenn bei einigen Aufführungen die Hauptdarstellerinnen bestrebt waren, zwei diametral 
entgegengesetzte Figuren darzustellen, von der Besetzung mit zwei Schauspielerinnen ganz zu schweigen. Bei der Verkörperung zweier Wesen der Protagonistin ging es Brecht nämlich nicht nur um die Verfremdung, die auf der Bühne statthat; in „der gute Mensch von Sezuan“ wird nicht weniger die Widersprüchlichkeit der menschlichen Natur in den Vordergrund gerückt. Seinem Wesen nach ist der Mensch gut, doch seine Umstände und Umgebung sind dergestalt, dass das Wohltun nur Verfall mit sich bringt, das Übeltun dagegen Wohlstand. Nachdem die Götter festgellt hatten, dass Shen Te der gute Mensch ist, stiegen sie wieder zum Himmel auf. Das Problem des Guten und des Bösen blieb dabei im Grunde genommen ungelöst. Ganz absichtlich lässt Bertolt Brecht das Ende des Stücks offen. Der Zuschauer des epischen Theaters soll selbst daraus eine Schlussfolgerung ziehen. Das offene Ende lässt eine gewisse Mehrdeutigkeit zu. Das Stück «Der gute Mensch von Sezuan» ist eine der besten Errungenschaften der epischen Dramendichtung Bertolt Brechts. Brecht versucht also vor allem an den Verstand zu appellieren und ein anschauliches Beispiel fürs Handeln aufzuführen. Wolf demgegenüber ist bestrebt, auf die Emotionen der Zuschauer zu wirken und mithilfe der Katharsis ihre Mitbeteiligung am Bühnengeschehen zu erlangen. In seinem Stück über Chinesin Tai Yang schlägt Wolf alle Wege der emotionalen Einwirkung auf den Zuschauer ein, die ihm zur Verfügung stehen. Zusammenfassend lässt sich festhalten: Die Vielfalt und der mehrdimensionale Ansatz zur Formulierung und Entwicklung des chinesischen Themas, je nach politischer, ethischer und ästhetischer Orientierung des Dramatikers zeigen das Problemausmaß der deutschen sozialistischen Drama dieser Zeit, ebenso wie die Produktivität der deutsch-russischen Kulturkontakte. Der internationale universelle Schwerpunkt, der in der russischen Literatur der Berichtsperiode und insbesondere im Konzept der Russischen Avantgarde und ihrer besten Vertreter, wie Sergej Tretjakow, zum Ausdruck kam, erwies sich außerordentlich anziehend für die deutschen sozialistischen Schriftsteller B. Brecht, I. Becher, F. Wolf und andere, die aus der „Schule“ des Expressionismus stammten. S. Tretjakow erwies sich als Persönlichkeit und als Künstler, als so eine hervorragende und vielseitige Individualität, dass die kreativen Kontakte zu ihm der Entwicklung des deutschen sozialistischen Dramas und vor allem der Theateraktivitäten von Bertolt Brecht und Friedrich Wolf einen starken Impuls gaben. 


\section{Список литературы}

Брехт Б. Ме-ти. Книга перемен // Брехт Б. Собрание избранных сочинений. М.: «Логос-Альтера», «Ессе homo», 2004. Т. г: Проза. 224 с. Вольф Ф. Годы и люди. М.: Прогресс, 1988. 374 с.

3 Гирин Ю.Н. Картина мира эпохи авангарда. Авангард как системная целостность. М.: ИМЛИ РАН, 2013. 400 с.

4 Головчинер B.E. Сергей Третьяков - учитель Бертольта Брехта // Сибирский филологический журнал. 20I4. № 2. С. II7-I25.

5 Гомолицкая-Третьякова Т.С. О моем отце // Третьяков С.М. Страна-перекресток. Документальная проза. М.: Сов. писатель, І99І. С. 554-564.

6 Земляной С. Этика Бертольта Брехта // Этическая мысль. М.: ИФ РАН, 2004. Вып. 5. С. 37-53.

7 Ленин В.И. Полн. собр. соч. М.: «ЁЁ Медиа», І970. Т. г. С. 440-44I.

8 Опиумные войны Китая. URL: http://www.shaolin.ru/china/history/ı8о (дата обращения: II.06.2019).

9 Первый Всесоюзный съезд советских писателей I934. Стенографический отчёт. Москва: ГИХЛ, І934. 7І 8 с. «Рычи, Китай!» Сергея Третьякова // Педагогика школьная. URL: http://www. portalus.ru/modules/shkola/rus_readme.php?archive=006\&id=I296I3I495\&start from=\&subaction=showfull\&ucat (дата обращения: ІІ.о6.20I9). SovLitСовЛит: Третьяков С. Рычи Китай. URL: http://www.ruthenia.ru/sovlit/j/2952.html (дата обращения: II.06.20I9). Третьяков С.М. Дэн Ши-хуа. Люди одного костра. Страна-перекресток. М.: Сов. писатель, г962. 770 с. Шарыпина T.A. Концепция «документального эпоса» русского авангарда и становление театральной практики Бертольта Брехта // Ученые записки Петрозаводского государственного университета. 20I6. № 3 (I56). С. 67-74.

I4 Шумахер Э. Жизнь Брехта. М.: Радуга, І988. 352 с.

I5 Brecht Bertolt. Der gute Mensch von Sezuan. Suhrkamp Verlag: Frankfurt a.M., I964. I $44 \mathrm{~S}$.

I6 Brecht Bertolt. Me-ti. Buch der Wendungen. Aufbau-Verlag: Berlin, I975. 260 S.

I7 Bertolt Brecht. Werke. Große kommentierte Berliner und Frankfurter Ausgabe. Herausgegeben von Werner Hecht, Jan Knopf, Werner Mittenzwei, Klaus-Detlef Müller. Band 26. Berthold Brecht Journale I I9I3-I94I: Tagebücher I9I3-I922. Journale I938-I94I. Autobiographische Notizen I9I9-194I Aufbau-Verl.. Brel. und Weimar; Suhrkamp Verl. Frankfurt am Main, I994. S. 326-327.

I8 Brecht-Lexikon. Ana Kugli / Michael Opitz (Hrsg.). Stuttgart-Weimar, 2006. 289 S.

I9 Jarmatz K., Barck S., Diczel P. Exil in der UDSSR. Leipzig, I979. 662 S.

20 KnopfJ. Bertolt Brecht. Lebenskunst in finsteren Zeiten. München: Carl Hanser Verlag, 2012.560 S. 
2I Lehmann J. Russische Literatur in Deutschland. Ihre Rezeption durch deutschsprachige Schriftsteller und Kritiker vom I8. Jahrhundert bis zur Gegenwart / Verlag J. B. Metzler, Stuttgart, 20I5. $432 \mathrm{~S}$.

22 Sharypina T., Ivlieva P. Das chinesische Tema in der deutschen Dramaturgie der dreißiger bis vierziger Jahre des 20. Jahrhunderts (Friedrich Wolf, Bertolt Brecht)// Akten des XIII. Kongresses der Internationalen Vereinigung für Germanistik (IVG), Shanghai 20I5. Germanistik zwischen Tradition und Innovation. Band 9. Frankfurt am M. etc.: Peter Lang. (= Publikationen der Internationalen Vereinigung für Germanistik 28). S. I43-I47.

23 Wolf F. Dramen. Berlin: Aufbau-Verlag, I960. 432 S. 


\section{References}

Brekht B. Me-ti. Kniga peremen [Book of Changes]. In: Brekht B. Sobranie izbrannykh sochinenii [Collection of selected works]. Moscow, "Logos-Al'tera”, "Esse homo” Publ., 2004. Vol. I: Proza [Prose]. 224 p. (In Russ.)

$2 \quad$ Vol'f F. Gody i liudi [Years and people]. Moscow, Progress Publ., I988. 374 p. (In Russ.)

3 Girin Iu.N. Kartina mira epokhi avangarda. Avangard kak sistemnaia tselostnost [The worldview of the epoch. Avant-garde as a systematic integrity]. Moscow, IWL RAS Publ., 20I3. 400 p. (In Russ.)

4 Golovchiner V.E. Sergei Tret'iakov - uchitel` Bertol'ta Brekhta [Sergey Tretyakov Bertolt Brecht's teacher]. Sibirskii filologicheskii zhurnal, 20I4, no 2, pp. II7-I25. (In Russ.) Gomolitskaia-Tret'iakova T.S. O moem ottse [About my father]. In: Tret'iakov S.M. Strana-perekrestok. Dokumental'naia proza [Country-intersection. Documentary prose]. Moscow, Sovetskii pisatel' Publ., I99I, pp. 554-564. (In Russ.) Zemlianoi S. Etika Bertol'ta Brekhta [Bertolt Brecht's ethics]. In: Eticheskaia mysl' [Ethical thought]. Moscow, IF RAN Publ., 2004, issue 5, pp. 37-53. ( In Russ.) Lenin V.I. Polnoe sobranie sochinenii [Complete works]. Moscow, "EE Media” Publ., I970, vol. I, pp. 440-44I. (In Russ.)

8 Opiumnye voiny Kitaia [Opium Chinese wars]. Available at: http://www.shaolin.ru/ china/history/I80 (Accessed II June 20I9). (In Russ.)

9 Pervyi Vsesoiuznyi s"ezd sovetskikh pisatelei 1934. Stenograficheskii otchet [The first all-Union Congress of Soviet writers in I934. Verbatim record]. Moscow, GIKhL Publ., I934. 7I8 p. (In Russ.)

“Rychi, Kitai!” Sergeia Tret‘iakova [Roar, China! by Sergey Tretyakov]. Pedagogika shkol'naia [School pedagogy]. Available at: http://www.portalus.ru/modules/shkola/ rus_readme.php?archive $=006 \&$ id $=$ I296I3I495\&start_from $=\&$ subaction $=$ showfull\&ucat (Accessed II June 20I9). (In Russ.) SovLitSovLit: Tret'iakov S. Rychi Kitai [Roar, China!]. Available at: http://www.ruthenia.ru/sovlit/j/2952.html (Accessed II June 20I9). (In Russ.) Tret'iakov S.M. Den Shi-khua. Liudi odnogo kostra. Strana-perekrestok [Dan Shih Hua. People of one bonfire. Cross country]. Moscow, Sovetskii pisatel' Publ., I962. 770 p. (In Russ.) Sharypina T.A. Kontseptsiia “dokumental'nogo eposa” russkogo avangarda i stanovlenie teatral'noi praktiki Bertol'ta Brekhta [Conceptual ideas of "documentary epos" in Russian avant-garde and the development of Bertolt Brecht's theatrical practice]. Uchenye zapiski Petrozavodskogo gosudarstvennogo universiteta, 20I6, no 3 (I56), pp. 67-74. (In Russ.)

I4 Shumakher E. Zhizn` Brekhta [The life of Brecht]. Moscow, Raduga Publ., I988. 352 p. (In Russ.) 
I5 Brecht Bertolt. Der gute Mensch von Sezuan. Frankfurt a.M., Suhrkamp Verlag, I964. I44 S. (In German)

I6 Brecht Bertolt. Me-ti. Buch der Wendungen. Berlin, Aufbau-Verlag, I975. 260 S. (In German)

I7 Bertolt Brecht Werke. Große kommentierte Berliner und Frankfurter Ausgabe. Herausgegeben von Werner Hecht, Jan Knopf, Werner Mittenzwei, Klaus-Detlef Müller. Band 26. Berthold Brecht Journale I I9I3-I94I: Tagebücher I9I3-I922. Journale I938-I94I. Autobiographische Notizen I919-I94I Aufbau-Verl. Brel. und Weimar; Suhrkamp Verl. Frankfurt am Main 1994. S. 326-327. (In German)

I8 Brecht-Lexikon. Ana Kugli / Michael Opitz (Hrsg.). Stuttgart-Weimar, 2006. 289 S. (In German)

I9 Jarmatz K., Barck S., Diczel P. Exil in der UDSSR. Leipzig, I979. 662 S. (In German)

20 Knopf J. Bertolt Brecht. Lebenskunst in finsteren Zeiten. München, Carl Hanser Verlag, 20I2. 560 S. (In German)

2I Lehmann J. Russische Literatur in Deutschland. Ihre Rezeption durch deutschsprachige Schriftsteller und Kritiker vom I8. Jahrhundert bis zur Gegenwart. Stuttgart, Verlag J.B. Metzler, 20I5. 432 S. (In German)

22 Sharypina T., Ivlieva P. Das chinesische Tema in der deutschen Dramaturgie der dreißiger bis vierziger Jahre des 20. Jahrhunderts (Friedrich Wolf, Bertolt Brecht). Akten des XIII. Kongresses der Internationalen Vereinigung für Germanistik (IVG), Shanghai 2015. Germanistik zwischen Tradition und Innovation. Band 9. Frankfurt am M. etc.: Peter Lang. (= Publikationen der Internationalen Vereinigung für Germanistik 28). S. I43-I46. (In German)

23 Wolf F. Dramen. Berlin, Aufbau-Verlag, I960. 432 S. (In German) 